\title{
Optimal beam arrangement for pulmonary ventilation image-guided intensity-modulated radiotherapy for lung cancer
}

\author{
Ruihao Wang ${ }^{1 \dagger}$, Shuxu Zhang ${ }^{1 *+}$, Hui Yu' ${ }^{1}$ Shengqu Lin ${ }^{1}$, Guoqian Zhang ${ }^{1}$, Rijie Tang ${ }^{2}$ and Bin Qi ${ }^{3}$
}

\begin{abstract}
Background: The principal aim of this study was to evaluate the feasibility of incorporating four-dimensional (4D)-computed tomography (CT)-based functional information into treatment planning and to evaluate the potential benefits of individualized beam setups to better protect lung functionality in patients with non-small cell lung cancer (NSCLC).
\end{abstract}

Methods: Peak-exhale and peak-inhale CT scans were carried out in 16 patients with NSCLC treated with intensity-modulated radiotherapy (IMRT). 4D-CT-based ventilation information was generated from the two sets of CT images using deformable image registration. Four kinds of IMRT plans were generated for each patient: two anatomic plans without incorporation of ventilation information, and two functional plans with ventilation information, using either five equally spaced beams (FESB) or five manually optimized beams (FMOB). The dosimetric parameters of the plans were compared in terms of target and normal tissue structures, with special focus on dose delivered to total lung and functional lung.

Results: In both the anatomic and functional plans, the percentages of both the functional and total lung regions irradiated at $V_{5}, V_{10}$, and $V_{20}$ (percentage volume irradiated to $>5,>10$ and $>20 \mathrm{~Gy}$, respectively) were significantly lower for FMOB compared with FESB $(P<0.05)$, but there was no significant difference for $V_{30}(P>0.05)$. Compared with FESB, a greater degree of sparing of the functional lung was achieved in functional IMRT plans with optimal beam arrangement, without compromising target volume coverage or the irradiated volume of organs at risk, such as the spinal cord, esophagus, and heart.

Conclusions: Pulmonary ventilation image-guided IMRT planning with further optimization of beam arrangements improves the preservation of functional lung in patients with NSCLC.

Keywords: Intensity-modulated radiotherapy, Pulmonary ventilation, Four-dimensional computed tomography, Plan optimization

\section{Background}

Radiation therapy (RT) plays a significant role in the curative treatment of surgically inoperable non-small cell lung cancer (NSCLC). Radiation pneumonitis (RP) is the most common complication of RT for NSCLC, and its occurrence and severity are closely correlated with the mean lung dose, total lung volume irradiated to $>20$ Gy $\left(\mathrm{V}_{20}\right)$, location of the tumor, pulmonary function, and

\footnotetext{
*Correspondence: gthzsx@163.com

${ }^{\dagger}$ Equal contributors

'Departments of Radiotherapy, Cancer Hospital of Guangzhou Medical University, 78 Hengzhigang Road, Guangzhou 510095, China

Full list of author information is available at the end of the article
}

simultaneous or sequential chemotherapy [1-4]. Graham et al. [5] showed that when the $V_{20}$ was $<22 \%$, the incidence of RP within 2 years was zero; however, the incidences of RP within 2 years increased to $7 \%, 13 \%$, and $36 \%$ when the $\mathrm{V}_{20}$ percentages were $22-31 \%, 32-40 \%$, and $>40 \%$, respectively. This risk of RP could be reduced by up to $10 \%$ by using intensity-modulated radiotherapy (IMRT), without compromising tumor-dose delivery [6]. Nevertheless, ways of minimizing RT-induced side effects such as $\mathrm{RP}$, while continuing to achieve reasonable local control of NSCLC, remain a challenge for radiation oncologists. 
Information on pulmonary function provided by perfusion and/or ventilation imaging has been shown to be important in evaluating pulmonary toxicity after RT for NSCLC [7-10]. Several techniques exist for pulmonary ventilation imaging, including single photon emission computed tomography and X-ray CT (SPECT-CT) $[7,8]$, hyperpolarized helium-3 magnetic resonance imaging $\left({ }^{3} \mathrm{He}-\mathrm{MRI}\right)[9,10]$, and inert xenon CT (Xe-CT) [11]. However, although all these imaging modalities can provide useful ventilation information, each has serious drawbacks, such as high cost, low resolution, long scan time and/or low accessibility [12].

Four-dimensional (4D) CT imaging is an exciting new form of ventilation imaging, which consists of threedimensional (3D) CT images resolved into different phases of the breathing cycle [13]. 4D-CT is becoming widely available and has shown great promise for treatment planning. Because 4D-CT data are relatively easy to acquire during treatment planning, calculating ventilation maps from 4D-CT data only involves additional image processing, and does not add any extra dosimetric or monetary cost to the patient. Moreover, 4D-CT ventilation imaging has higher resolution, a shorter scan time and is more accessible than other existing techniques. Previous studies have investigated the incorporation of 4D-CT ventilation imaging into RT treatment planning [8,14-16]. For example, Castillo et al. [8] examined different ways of calculating ventilation from $4 \mathrm{D}-\mathrm{CT}$ data to estimate local volume changes, and compared the results with those obtained clinically from SPECT ventilation. Ding et al. [14] explored the changes in lung ventilation after RT, and Yamamoto et al. [15] and Yaremko et al. [16] discussed the idea of designing treatment plans to avoid high-ventilation areas of the lung. However, most of these studies considered treatment planning based on fixed-beam arrangements, and to the best of our knowledge, no studies have compared the use of different beam arrangements.

The primary purpose of this study was to investigate the feasibility of combining IMRT with ventilation maps calculated from 4D-CT data, and to compare the benefits of optimal and fixed-beam arrangements in IMRT for NSCLC.

\section{Methods}

\section{Patient selection}

This study was approved by the Institutional Review Board (IRB) at the Affiliated Tumor Hospital of Guangzhou Medical University. Informed consent was obtained from each patient, in accordance with the IRB regulations.

Sixteen consecutive patients with Stage III-IV and recurrent NSCLC who previously underwent IMRT were included. The characteristics of the patients are presented in Table 1.The 4D-CT data sets were obtained using a 16-detector row spiral CT scanner (Lightspeed 16; GE
Table 1 Patient characteristics

\begin{tabular}{|c|c|}
\hline Characteristic & n (\%) \\
\hline \multicolumn{2}{|l|}{ Age (yr) } \\
\hline median & 55 \\
\hline range & $38-73$ \\
\hline \multicolumn{2}{|l|}{ Sex } \\
\hline male & $14(87.5)$ \\
\hline female & $2(12.5)$ \\
\hline \multicolumn{2}{|l|}{ Histologic type } \\
\hline NSCLC, adenocarcinoma & $5(31.2)$ \\
\hline NSCLC, squamous cell carcinoma & $11(68.8)$ \\
\hline \multicolumn{2}{|l|}{ Stage } \\
\hline IIIA & $3(18.8)$ \\
\hline$\| \mathrm{B}$ & $4(25.0)$ \\
\hline IV & $7(43.7)$ \\
\hline Recurrent & $2(12.5)$ \\
\hline \multicolumn{2}{|l|}{ Location } \\
\hline RUL & $4(25.0)$ \\
\hline RLL & $5(31.2)$ \\
\hline LUL & $4(25.0)$ \\
\hline LLL & $3(18.8)$ \\
\hline \multicolumn{2}{|l|}{$\operatorname{PTV}\left(\mathrm{cm}^{3}\right)$} \\
\hline median & 596 \\
\hline range & $176-3647$ \\
\hline
\end{tabular}

Abbreviations: NSCLC Non-small cell lung cancer, RUL Right upper lobe, RLL Right lower lobe, LUL Left upper lobe, LLL Left lower lobe, PTV Planning target volume.

Medical Systems, Milwaukee, WI, USA) in cine mode with a $2.5-\mathrm{mm}$ slice covering the entire chest. A medical physicist was present during each CT scan to ensure that the patient's breathing was not erratic. To compute the pulmonary ventilation, the paired $4 \mathrm{D}-\mathrm{CT}$ images at the peak-exhale and peak-inhale phases were selected in this study. Additional details regarding the use of 4D-CT have been published in our previous work [17].

\section{D-CT pulmonary ventilation imaging}

Processing 4D-CT pulmonary ventilation imaging involved three steps. Firstly, paired 4D-CT images obtained at the peak-exhale and peak-inhale phases were transferred to our self-developed ventilation imaging software (ZHANGShuxu 4D-CT LF, V1.0) for ventilation computation. Deformable image registration (DIR) was then used to link corresponding lung volume elements between the peak-exhale and peak-inhale 4D-CT data sets, to produce a displacement vector field (DVF). We used 3D-image registration based on a B-spline elastic algorithm, the geometric accuracy of which has been validated previously [18]. 3D-image registration was used to create a voxel-by-voxel displacement map showing the motion of the lung tissue as a function 
of respiratory state. Finally, a 4D-CT ventilation image was created by quantitative analysis of the DVF. In this study, the Jacobian determinant of deformation [19] was used to measure the degree of regional lung expansion that was directly related to specific volume change. The Jacobian determinant of DVF was calculated for each voxel in the lung and used to represent local tissue expansion (or contraction). The regional ventilation was given by:

$$
V(x, y, z)=\left|\begin{array}{ccc}
1+\frac{\partial u_{x}(x, y, z)}{\partial x} & \frac{\partial u_{x}(x, y, z)}{\partial y} & \frac{\partial u_{x}(x, y, z)}{\partial z} \\
\frac{\partial u_{y}(x, y, z)}{\partial x} & \frac{1+\partial u_{y}(x, y, z)}{\partial y} & \frac{\partial u_{y}(x, y, z)}{\partial z} \\
\frac{\partial u_{z}(x, y, z)}{\partial x} & \frac{\partial u_{y}(x, y, z)}{\partial y} & \frac{1+\partial u_{z}(x, y, z)}{\partial z}
\end{array}\right|-1
$$

where the function represents the displacement vector, mapping the voxel at each point of a peak-exhale image to the corresponding position on a peak-inhale image. In

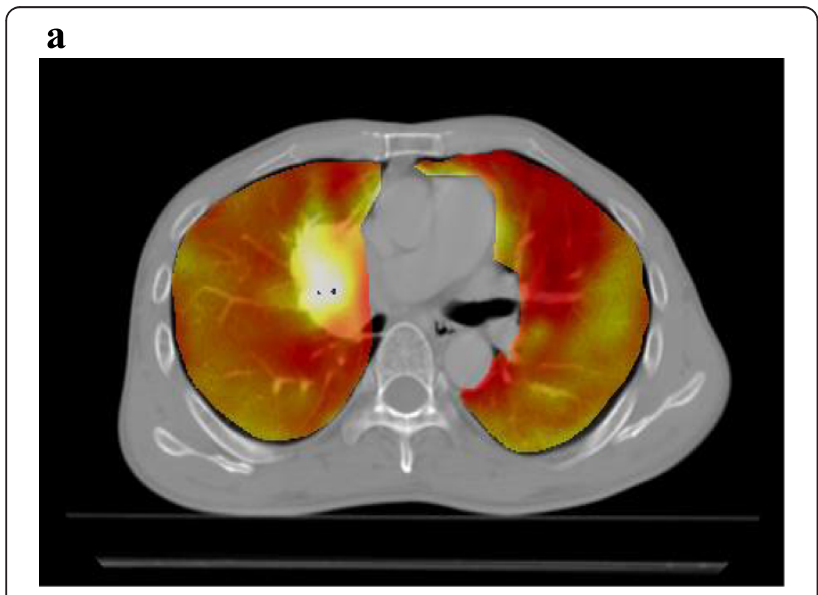

b

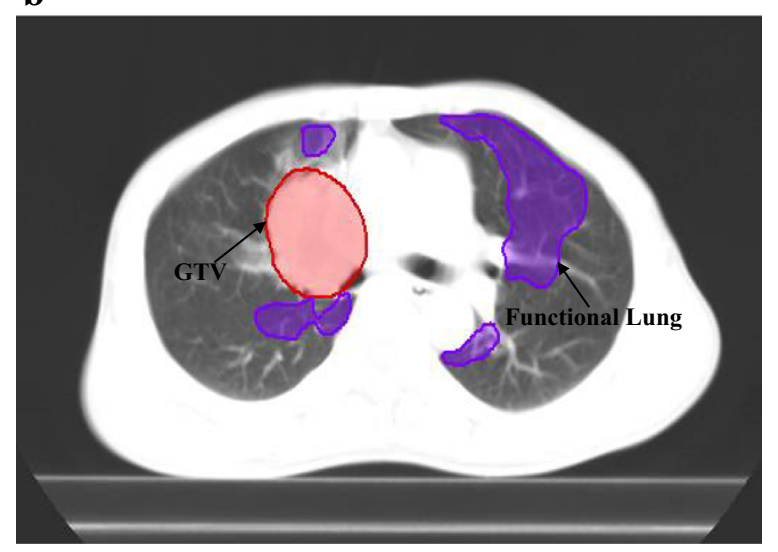

Figure 1 The functional lung of ventilation maps in a case. (a) Fusion imaging of $\mathrm{CT}$ image and pulmonary ventilation extracted from the generated deformation field; and (b) highly functional lung regions contoured on (a). In this study, regions in the top 30\% for ventilation in the ventilation image were defined as functional lung structures to avoid in the functional IMRT plans. addition, the functional lung of ventilation mapping procedure was shown in Figure 1.

\section{IMRT planning}

The Oncentra treatment planning system, version 4.1 (Nucletron V.B., Veenendaal, The Netherlands) was used to delineate the target volumes and organs at risk (OARs) and for IMRT planning and dose calculation. Both target volumes and OARs, including the spinal cord, esophagus, heart, and lungs, were contoured and approved on peak-exhale 4D-CT images by an attending physician. The gross tumor volume to clinical target volume (CTV), and CTV to planning target volume (PTV) margins were $6-8 \mathrm{~mm}$ and $8 \mathrm{~mm}$, respectively. Planning OAR volumes were generated for the spinal cord and esophagus by adding $5.0-\mathrm{mm}$ isotropic margins. In addition, functional lung regions were defined from 4D-CT ventilation images for functional planning, as described for other IMRT studies for NSCLC [16]. In this study, regions in the top $30 \%$ for ventilation in the 4D-CT-based ventilation image were set as functional lung structures to avoid in the functional IMRT plans. Moreover, the total lung and functional lung represented the total volume and the functional part of the sum of both lungs.

All the IMRT plans were designed to deliver 66 Gy in 33 fractions to cover $95 \%$ of the PTV. The radiation dose distributions were calculated using the collapsed cone algorithm on a graphics processing unit (GPU). Four independent treatment plans were designed for each case in this study. First, two anatomically-constrained plans was generated without the incorporation of pulmonary ventilation information, with the main goal of minimizing the

\section{Table 2 IMRT planning goals and constraints}

\begin{tabular}{ll}
\hline Structure & Constraint \\
\hline PTV & Min. dose $\geq 65$ Gy \\
& Max. dose $\leq 70$ Gy \\
& Volume receiving $\geq 66$ Gy more than $95 \%$ \\
& Dean 20 Gy \\
Total lung & Volume receiving $\geq 20$ Gy less than $30 \%$ \\
& Volume receiving $\geq 30$ Gy less than $20 \%$ \\
Spinal cord & Max. point dose $\leq 40$ Gy \\
Heart & Volume receiving $\geq 40$ Gy less than $100 \%$ \\
& Volume receiving $\geq 45$ Gy less than $67 \%$ \\
& Volume receiving $\geq 60$ Gy less than $33 \%$ \\
Esophagus & Volume receiving $\geq 55$ Gy less than $35 \%$ \\
Functional lung & Max. dose $\leq 70$ Gy \\
\hline
\end{tabular}

Abbreviations: IMRT Intensity-modulated radiotherapy, PTV Planning target volume, Min. Minimum, Max. Maximum, $D_{\text {mean }}$ Mean dose. ${ }^{a}$ for functional-constrained plan. 
Table 3 Comparison of PTV parameters between different plans

\begin{tabular}{|c|c|c|c|c|c|c|}
\hline \multirow[b]{2}{*}{ Paremeter } & \multicolumn{3}{|c|}{ Anatomic plan } & \multicolumn{3}{|c|}{ Functional plan } \\
\hline & FESB & FMOB & $P$ value & FESB & FMOB & $P$ value \\
\hline Conformity index ${ }^{a}$ & $1.01 \pm 0.01$ & $1.013 \pm 0.02$ & 0.148 & $1.01 \pm 0.02$ & $1.02 \pm 0.02$ & 0.617 \\
\hline Homogeneity index ${ }^{\mathrm{b}}$ & $1.06 \pm 0.02$ & $1.07 \pm 0.03$ & 0.069 & $1.07 \pm 0.02$ & $1.07 \pm 0.03$ & 0.837 \\
\hline MUs & $485 \pm 202$ & $497 \pm 207$ & 0.403 & $492 \pm 189$ & $500 \pm 223$ & 0.682 \\
\hline Mean dose (Gy) & $67.11 \pm 0.05$ & $67.18 \pm 0.54$ & 0.637 & $67.09 \pm 0.40$ & $67.04 \pm 0.42$ & 0.785 \\
\hline
\end{tabular}

Abbreviations: PTV Planning target volume, MUs Monitor units, FESB Five equally-spaced beams, FMOB Five manually optimized beams; Data presented as mean \pm standard deviation.

${ }^{a}$ Conformity index $=$ cover factor $\times$ spill factor, where cover factor defined as relative PTV volume receiving $\geq 66$ Gy, and spill factor defined as ratio of PTV receiving $\geq 66$ Gy to total volume receiving $66 \mathrm{~Gy}$.

${ }^{b}$ Homogeneity index $=D_{5 \%} / D_{95 \%}$, where $D_{x \%}$ is minimum dose in $x \%$ of PTV.

volumes of total normal lung and other normal tissues that were irradiated above their tolerance levels (Table 2). Second, two functionally-constrained plans incorporating pulmonary ventilation information was generated with additional constraints to reduce the $V_{10}$ and $V_{20}$ of the identified functional lung regions (Table 2). In addition, dosimetric differences between beam arrangements were evaluated using five equally spaced beams (FESB) positioned every $72^{\circ}$ from the anterior and lateral directions, and five manually optimized beams (FMOB) for both the anatomic and functional plans. In plan with FMOB, gantry angles were changed to avoid critical OARs, highly functional lung regions and the non-involved total lung regions.

\section{Data analysis and statistical methods}

The quality of the IMRT plans was evaluated by comparing the metrics of PTVs using common dose-volume parameters such as the conformity index $(\mathrm{CI})$, heterogeneity index $(\mathrm{HI})$, mean dose $\left(\mathrm{D}_{\text {mean }}\right)$, and total number of monitor units (MUs). Dosimetric parameters including the percentage of lung volume receiving $>5 \mathrm{~Gy},>10 \mathrm{~Gy},>20 \mathrm{~Gy}$, and $>30$ Gy were calculated for the functional lung $\left(\mathrm{FLV}_{5}, \mathrm{FLV}_{10}, \mathrm{FLV}_{20}\right.$, and $\mathrm{FLV}_{30}$, respectively) and total lung $\left(\mathrm{TLV}_{5}, \mathrm{TLV}_{10}\right.$, $T L V_{20}$, and $T L V_{30}$, respectively). Regarding the other critical structures, the maximum dose $\left(D_{\max }\right)$ delivered to the spinal cord, the $D_{\text {mean }}$ and $D_{\max }$ delivered to esophagus, and the $\mathrm{V}_{40}, \mathrm{~V}_{45}$, and $\mathrm{V}_{60}$ delivered to the heart were also quantified and compared between the anatomic and functional plans with different beam arrangements.

Statistical analysis was performed using SPSS 19.0 statistical software (SPSS Inc., Chicago, IL, USA). Volumetric and dosimetric parameters for the target volumes and OARs were characterized using descriptive statistics. The dosimetric differences between the application of FESB and FMOB were evaluated for each plan and compared using paired, two tailed $t$-tests. A P value $<0.05$ was considered statistically significant.

\section{Results}

All the anatomically-constrained and functional-constrained plans developed in this study were clinically acceptable. There were no significant differences in PTV dosimetric parameters such as $\mathrm{CI}, \mathrm{HI}$, and $\mathrm{D}_{\text {mean }}(\mathrm{P}>0.05)$ between the anatomic and functional plans using FMOB or FESB, as shown in Table 3. In addition, the functional plans resulted in slightly higher MUs than the anatomic plans, as expected given the additional constraints for functional planning, though the differences were not significant.

Table 4 shows the dosimetric parameters of the total and functional lung for the same beam arrangements between anatomic and functional plan. For FESB, functional

Table 4 Comparison of dosimetric parameters for total and functional lung between anatomic and functional plan for the same beam setups

\begin{tabular}{|c|c|c|c|c|c|c|c|c|c|}
\hline \multirow[t]{2}{*}{ Beam setup } & \multirow[b]{2}{*}{ Plan } & \multicolumn{4}{|l|}{ Total lung } & \multicolumn{4}{|c|}{ Functional lung } \\
\hline & & $\mathrm{TLV}_{5}$ & $\operatorname{TLV}_{10}$ & $\mathrm{TLV}_{20}$ & $\mathrm{TLV}_{30}$ & $\mathrm{FLV}_{5}$ & $\mathrm{FLV}_{10}$ & $\mathrm{FLV}_{20}$ & $\mathrm{FLV}_{30}$ \\
\hline \multirow[t]{3}{*}{ FESB } & Anatomic plan & $61.7 \pm 12.9$ & $46.7 \pm 11.4$ & $30.7 \pm 5.3$ & $21.2 \pm 5.3$ & $54.2 \pm 21.7$ & $42.5 \pm 22.1$ & $26.3 \pm 20.7$ & $17.0 \pm 12.6$ \\
\hline & Functional plan & $60.3 \pm 12.8$ & $43.5 \pm 11.3$ & $29.1 \pm 6.7$ & $20.9 \pm 5.6$ & $52.4 \pm 20.7$ & $37.1 \pm 12.2$ & $21.1 \pm 5.8$ & $16.7 \pm 6.8$ \\
\hline & $P$ value & 0.002 & 0.001 & 0.051 & 0.232 & 0.005 & 0.029 & 0.018 & 0.911 \\
\hline \multirow[t]{3}{*}{ FMOB } & Anatomic plan & $35.0 \pm 12.5$ & $29.1 \pm 9.8$ & $23.3 \pm 7.1$ & $20.1 \pm 6.4$ & $30.2 \pm 18.5$ & $24.1 \pm 11.5$ & $17.8 \pm 7.5$ & $16.2 \pm 7.0$ \\
\hline & Functional plan & $33.3 \pm 10.1$ & $27.6 \pm 8.5$ & $23.3 \pm 6.9$ & $20.1 \pm 6.1$ & $29.2 \pm 18.3$ & $23.0 \pm 14.8$ & $16.7 \pm 9.7$ & $14.7 \pm 9.5$ \\
\hline & $P$ value & 0.395 & 0.226 & 0.945 & 0.904 & 0.757 & 0.575 & 0.234 & 0.258 \\
\hline
\end{tabular}

Abbreviations: TLVx Percentage of volume of total lung receiving > x Gy, FLVx Percentage of volume of functional lung receiving > $x$ Gy, FESB Five equally-spaced beams, FMOB Five manually optimized beams, Data presented as mean \pm standard deviation. 

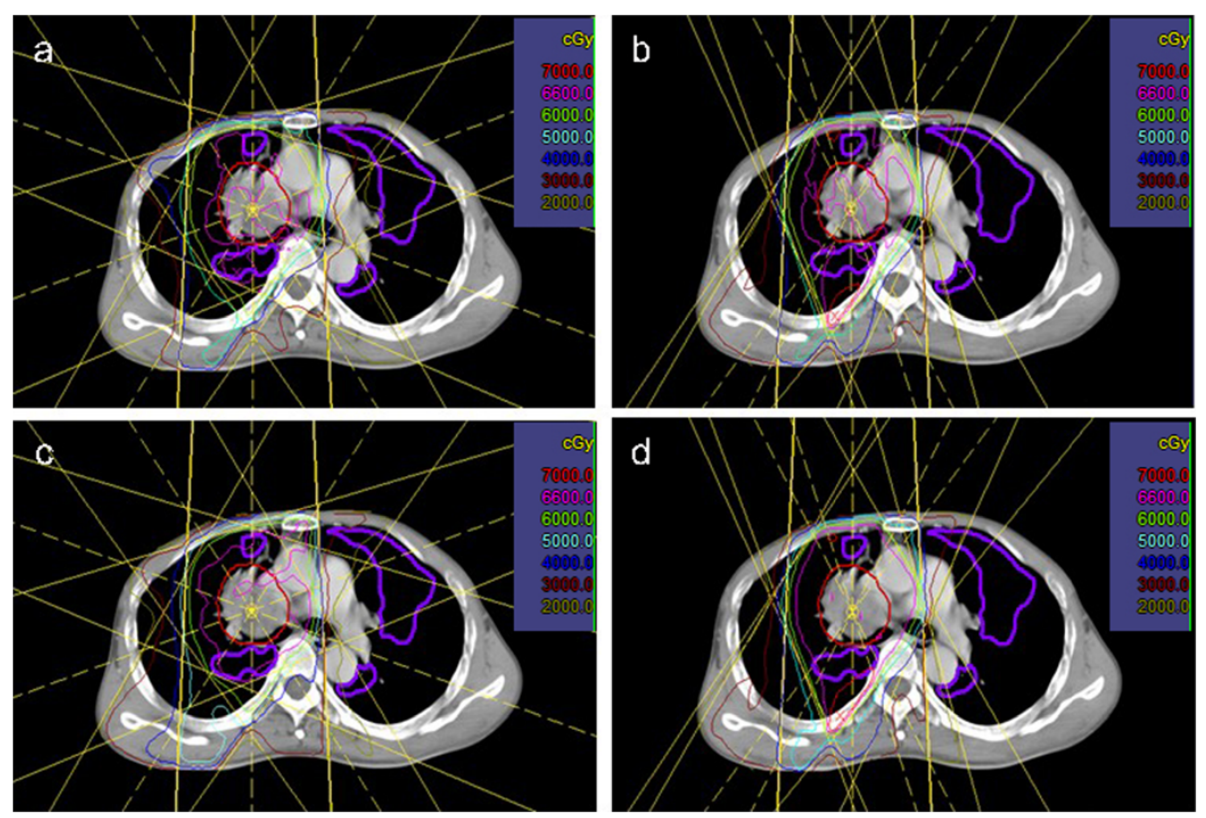

Figure 2 Comparison of the dose distribution between an anatomic plan with (a) five equally-spaced beam arrangements; and (b) five manually optimized beam arrangements; a functional plan with (c) five equally-spaced beam arrangements; and (d) five manually optimized beam arrangements.

plans had lower doses irradiated to the total and functional lung in average compared with those in anatomic plans, with significant reductions $(\mathrm{P}<0.05)$ in lower dose region $\left(\mathrm{V}_{5}, \mathrm{~V}_{10}\right.$, and $\left.\mathrm{V}_{20}\right)$; While for $\mathrm{FMOB}$, functional plans resulted in slightly lower doses to the total and functional lung than those in anatomic plans, though the differences were not statistically significant $(P>0.05)$.

Figure 2 shows the isodose distributions for both anatomic and functional plans and for both beam arrangements as an example. Both anatomic and functional plans showed that the highly functional lungs were spared in FMOB, compared with FESB. In particular, the 20- and $30-G y$ isodose curves were significantly distorted and moved out of the left lung, which contained large part of highly functional lung. The dosimetric parameters for the total and functional lung regions between different beam arrangements are shown in Tables 5 and 6 . In both the anatomic and functional plans, $\mathrm{V}_{5}, \mathrm{~V}_{10}$, and $\mathrm{V}_{20}$ in the total and functional lung regions were significantly lower in FMOB, compared with FESB $(\mathrm{P}<0.05)$, though there was no significant difference for $\mathrm{V}_{30}(\mathrm{P}>0.05)$. The same results were demonstrated using a dose-volume histogram (DVH) (Figure 3).

The doses to the spinal cord, heart, and esophagus using the different beam arrangements are shown in Table 7. The maximum doses delivered to the spinal cord in the anatomic and functional plans were slightly lower for FMOB compared with FESB, but the difference was not significant $(\mathrm{P}>0.05)$. However, the doses delivered to the esophagus were significantly lower for FMOB than for FESB $(\mathrm{P}<0.05)$; the $\mathrm{D}_{\text {mean }}$ values for the esophagus in the anatomic plan were 22.0 Gy and 16.3 Gy for FESB and FMOB, respectively, while the equivalent $D_{\text {mean }}$ values in the functional plan were 22.7 Gy and $16.2 \mathrm{~Gy}$. In the heart, there were significant differences between the FESB and FOMB plans in terms of $\mathrm{V}_{60}$ for both the anatomic and functional plans $(\mathrm{P}<0.05)$, but the differences were not significant for $\mathrm{V}_{40}$ and $\mathrm{V}_{45}(\mathrm{P}>0.05)$.

Table 5 Comparison of dosimetric parameters for total lung between different beam arrangements

\begin{tabular}{|c|c|c|c|c|c|c|c|c|}
\hline \multirow[t]{2}{*}{ Beam arrangement } & \multicolumn{4}{|c|}{ Anatomic plan } & \multicolumn{4}{|c|}{ Functional plan } \\
\hline & $\operatorname{TLV}_{5}$ & $\mathrm{TLV}_{10}$ & $\mathrm{TLV}_{20}$ & $\mathrm{TLV}_{30}$ & $\operatorname{TLV}_{5}$ & $\operatorname{TLV}_{10}$ & $\mathrm{TLV}_{20}$ & $\mathrm{TLV}_{30}$ \\
\hline FESB & $61.7 \pm 12.9$ & $46.7 \pm 11.4$ & $30.7 \pm 5.3$ & $21.2 \pm 5.3$ & $60.3 \pm 12.8$ & $43.5 \pm 11.3$ & $29.1 \pm 6.7$ & $20.9 \pm 5.6$ \\
\hline FMOB & $35.0 \pm 12.5$ & $29.1 \pm 9.8$ & $23.3 \pm 7.1$ & $20.1 \pm 6.4$ & $33.3 \pm 10.1$ & $27.6 \pm 8.5$ & $23.3 \pm 6.9$ & $20.1 \pm 6.1$ \\
\hline$P$ value & $<0.001$ & $<0.001$ & $<0.001$ & 0.417 & $<0.001$ & $<0.001$ & $<0.001$ & 0.231 \\
\hline
\end{tabular}

Abbreviations: TLVx Percentage of volume of total lung receiving > x Gy, FESB Five equally-spaced beams, FMOB Five manually optimized beams, Data presented as mean \pm standard deviation. 
Table 6 Comparison of dosimetric parameters for functional lung between different beam arrangements

\begin{tabular}{|c|c|c|c|c|c|c|c|c|}
\hline \multirow[t]{2}{*}{ Beam arrangement } & \multicolumn{4}{|c|}{ Anatomic plan } & \multicolumn{4}{|c|}{ Functional plan } \\
\hline & $\mathrm{FLV}_{5}$ & $\mathrm{FLV}_{10}$ & $\mathrm{FLV}_{20}$ & $\mathrm{FLV}_{30}$ & $\mathrm{FLV}_{5}$ & $\mathrm{FLV}_{10}$ & $\mathrm{FLV}_{20}$ & $\mathrm{FLV}_{30}$ \\
\hline FESB & $54.2 \pm 21.7$ & $42.5 \pm 22.1$ & $26.3 \pm 20.7$ & $17.0 \pm 12.6$ & $52.4 \pm 20.7$ & $37.1 \pm 12.2$ & $21.1 \pm 5.8$ & $16.7 \pm 6.8$ \\
\hline FMOB & $30.2 \pm 18.5$ & $24.1 \pm 12.0$ & $17.8 \pm 8.9$ & $16.2 \pm 7.0$ & $29.2 \pm 18.3$ & $23.0 \pm 14.8$ & $16.7 \pm 9.7$ & $14.7 \pm 9.5$ \\
\hline$P$ value & $<0.001$ & $<0.001$ & 0.049 & 0.754 & $<0.001$ & $<0.001$ & 0.001 & 0.244 \\
\hline
\end{tabular}

Abbreviations: FLVX Percentage of volume of functional lung receiving > x Gy, FESB Five equally-spaced beams, FMOB Five manually optimized beams, Data presented as mean \pm standard deviation.

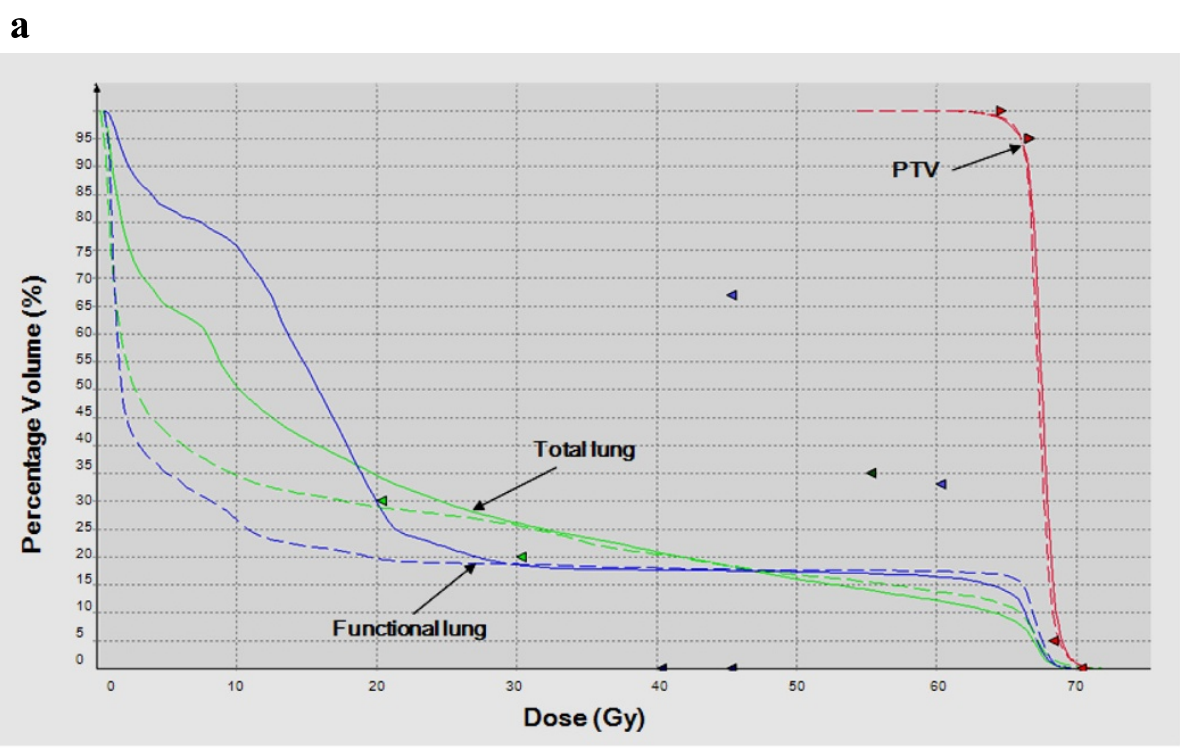

$\mathbf{b}$

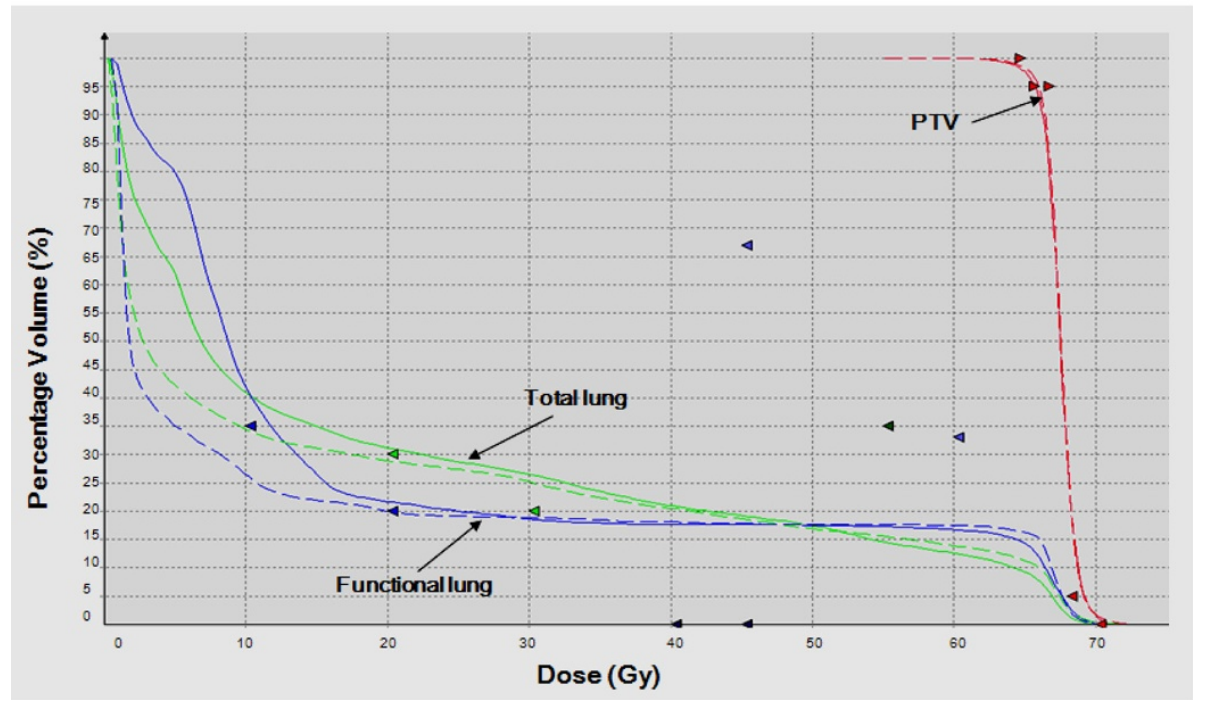

Figure 3 Dose-volume histogram (DVH) changes in planning target volume (PTV), total lung, and functional lung in a sample case.

(a) DVH of an anatomic-constrained IMRT treatment plan with five equally-spaced beam arrangements (solid lines) and five manual optimization beam arrangements (broken lines). (b) DVH changes are also shown in a functional-constrained IMRT plan using highly functional lung as an avoidance structure with different beam arrangements. 
Table 7 Comparison of dosimetric parameters for OARs between different beam arrangements

\begin{tabular}{|c|c|c|c|c|c|c|}
\hline \multirow[t]{2}{*}{ Parameter } & \multicolumn{3}{|c|}{ Anatomic plan } & \multicolumn{3}{|c|}{ Functional plan } \\
\hline & FESB & FMOB & $P$ value & FESB & FMOB & $P$ value \\
\hline \multicolumn{7}{|l|}{ Spinal cord } \\
\hline$D_{\max }(G y)$ & $37.1 \pm 7.1$ & $32.7 \pm 13.3$ & 0.101 & $37.1 \pm 7.8$ & $33.0 \pm 7.0$ & 0.113 \\
\hline \multicolumn{7}{|l|}{ Esophagus } \\
\hline $\mathrm{D}_{\text {mean }}(\mathrm{Gy})$ & $22.0 \pm 10.4$ & $16.3 \pm 11.6$ & 0.001 & $22.7 \pm 10.3$ & $16.2 \pm 11.6$ & 0.001 \\
\hline$D_{\max }(G y)$ & $58.1 \pm 9.6$ & $53.4 \pm 12.0$ & 0.013 & $57.6 \pm 10.4$ & $53.2 \pm 12.4$ & 0.020 \\
\hline \multicolumn{7}{|l|}{ Heart } \\
\hline$V_{40}$ & $17.2 \pm 11.1$ & $21.7 \pm 16.3$ & 0.144 & $17.2 \pm 11.7$ & $20.9 \pm 15.7$ & 0.167 \\
\hline$V_{45}$ & $13.1 \pm 9.1$ & $17.9 \pm 13.5$ & 0.057 & $13.4 \pm 10.1$ & $16.3 \pm 13.0$ & 0.194 \\
\hline$V_{60}$ & $5.9 \pm 4.6$ & $10.3 \pm 9.8$ & 0.032 & $5.8 \pm 5.0$ & $9.7 \pm 9.9$ & 0.048 \\
\hline
\end{tabular}

Abbreviations: OARs Organs at risk, $D_{\max }$ Maximum dose, $D_{\text {mean }}$ Mean dose, $V x$ Percentage of volume receiving $>x$ Gy, FESB Five equally-spaced beams, $F M O B$ Five manually optimized beams, Data presented as mean \pm standard deviation.

\section{Discussion}

Current RT planning aimed at limiting lung toxicity assumes a uniform distribution of pulmonary function, and fails to take account of spatial and temporal patterns. Previous studies demonstrated that the incidence and seriousness of RP were positively correlated with total radiation dose to the lungs [20]. Clarification of the relationship between radiation dose and changes in pulmonary function may help to predict and reduce RT-induced pulmonary toxicity. Decreasing the radiation dose to functional lung areas and directing the rays to the parts with perfusion/ventilation defects may help to protect highly functional lung regions and thus reduce the incidence and seriousness of RP [21].

Lung function can be evaluated by functional imaging modalities such as SPECT, PET/CT and ${ }^{3} \mathrm{He}$-MRI. However, these imaging modalities are not yet widely available in China, and their routine application in clinical practice would be associated with a long scan time and high costs to patients. We therefore assessed the value of acquiring functional information using the newly-reported 4D-CTbased ventilation imaging, which has the advantage that ventilation images can be calculated using only an additional processing step (i.e., DIR).

The results of this study showed that 4D-CT-based ventilation information could be used to reduce the radiation dose to the highly functional areas of the lung, assessed by $\mathrm{FLV}_{5-20}$, compared with anatomic planning alone, which was consistent with the results reported in previous published papers [15,16]. Moreover, our findings indicated that both anatomic and functional treatment plans using FMOB could reduce the functional lung regions exposed to lower doses $\left(\mathrm{FLV}_{5-20}\right)$, without compromising target volume coverage, compared with FESB. In addition to the observed improvements in dose-volume parameters of the functional lung, this study also identified dosimetric improvements in the low-dose zones of total lung, especially in terms of improved $V_{20}$ volume.
These results were compatible with that of a recent study investigating functional IMRT treatment planning using 4D-CT images [22]. Functional plans using an optimal beam arrangement aimed at preserving the functional lung did not compromise the DVHs of OARs, such as the spinal cord, esophagus, and heart, which may be an additional important clinical factor. These results also demonstrated that this technique could be applied safely to RT treatments for patients with NSCLC, without exceeding the dose-volume tolerances of OARs.

Although significant dosimetric improvements were observed in the lower-dose regions of functional and total lung, the clinical relevance of these improvements in terms of reducing the risk of RT-induced pulmonary toxicity remains unclear. There is currently insufficient outcome data to confirm the correlation between functional lung dose-volume parameters and pulmonary toxicity endpoints, and further studies are needed to determine if dosimetric reductions to functional lung will translate into clinical benefits for NSCLC patients.

Although, several existing studies have validated the 4D-CT-based ventilation imaging modality [23,24], its regional physiologic accuracy has not been validated in patients. In addition, temporal changes in regional ventilation to a segment of lung previously impaired by compression from a local tumor might occur during the course of RT. A possible explanation of these changes is that the shrinkage of lung tumor volume in response to IMRT treatment may result in increased ventilation as a result of reopening of the airways [25].

Ongoing studies aim to address the above issues by long-term follow-up of a preliminary patient cohort with known functional lung dose-volume parameters. The physiologic accuracy of 4D-CT-based ventilation imaging will be assessed in these patients by quantifying the impact of temporal changes in ventilation during the course of RT and evaluating pulmonary function and morbidity outcomes after radical RT. 


\section{Conclusions}

In conclusion, this study demonstrated the feasibility of functional treatment planning using 4D-CT-based pulmonary ventilation information to identify structures to avoid. The results further indicated the dosimetric benefit of optimal beam arrangement compared with fixed-beam arrangement in IMRT treatment planning, in terms of preserving functional lung at lower radiation doses in patients with NSCLC.

\section{Competing interests}

The authors declare that they have no competing interest.

\section{Authors' contributions}

SZ and SL conceived and designed the experiments. RW and GZ performed the experiments. RW, $\mathrm{HY}, \mathrm{RJ}$ and $\mathrm{BQ}$ collected the data and performed the statistical analysis. RW and SZ wrote the manuscript. All authors read and approved the final manuscript.

\section{Acknowledgements}

This study was partially supported by a National Natural Scientific Foundation of China grant (81170078), a Guangdong Provincial Science and Technology Agency grant (2011B031800111), and a Guangzhou Municipal Science and Technology Agency grant (2011 J4300131).

\section{Author details}

'Departments of Radiotherapy, Cancer Hospital of Guangzhou Medical University, 78 Hengzhigang Road, Guangzhou 510095, China. ${ }^{2}$ Departments of Radiology, Cancer Hospital of Guangzhou Medical University, 78 Hengzhigang Road, Guangzhou 510095, China. ${ }^{3}$ Departments of Radiation Oncology, Cancer Hospital of Guangzhou Medical University, 78 Hengzhigang Road, Guangzhou 510095, China.

Received: 10 October 2013 Accepted: 7 August 2014

Published: 16 August 2014

\section{References}

1. Mazeron R, Etienne-Mastroianni B, Perol D, Arpin D, Vincent M, Falchero L, Martel-Lafay I, Carrie C, Claude L: Predictive factors of late radiation fibrosis: a prospective study in non-small cell lung cancer. Int I Radiat Oncol Biol Phys 2010, 77:38-43.

2. Ding X, Ji W, Li J, Zhang X, Wang L: Radiation recall pneumonitis induced by chemotherapy after thoracic radiotherapy for lung cancer. Radiat Oncol 2011, 6:24.

3. Mehta V: Radiation pneumonitis and pulmonary fibrosis in non-small-cell lung cancer: pulmonary function, prediction, and prevention. Int I Radiat Oncol Biol Phys 2005, 63:5-24

4. Park YH, Kim JS: Predictors of radiation pneumonitis and pulmonary function changes after concurrent chemoradiotherapy of non-small cell lung cancer. Radiat Oncol J 2013, 31:34-40.

5. Graham MV, Purdy JA, Emami B, Harms W, Bosch W, Lockett MA, Perez CA: Clinical dose-volume histogram analysis for pneumonitis after $3 \mathrm{D}$ treatment for non-small cell lung cancer (NSCLC). Int J Radiat Oncol Biol Phys 1999, 45:323-329.

6. Murshed H, Liu HH, Liao Z, Barker JL, Wang X, Tucker SL, Chandra A, Guerrero T, Stevens C, Chang JY, Jeter M, Cox JD, Komaki R, Mohan R: Dose and volume reduction for normal lung using intensity-modulated radiotherapy for advanced-stage non-small-cell lung cancer. Int J Radiat Oncol Biol Phys 2004, 58(4):1258-1267.

7. Shioyama Y, Jang SY, Liu HH, Guerrero T, Wang X, Gayed IW, Erwin WD, Liao Z, Chang JY, Jeter M, Yaremko BP, Borghero YO, Cox JD, Komaki R, Mohan R: Preserving functional lung using perfusion imaging and intensity-modulated radiation therapy for advanced-stage non-small cell lung cancer. Int J Radiat Oncol Biol Phys 2007, 68:1349-1358.

8. Castillo R, Castillo E, Martinez J, Guerrero T: Ventilation from four-dimensional computed tomography: density versus Jacobian methods. Phys Med Biol 2010, 55:4661-4685.
9. van Beek EJR, Wild JM, Kauczor H-U, Schreiber W, Mugler JP, de Lange EE: Functional MRI of the lung using hyperpolarized 3-helium gas. J Magn Reson Imaging 2004, 20:540-554.

10. Fain S, Schiebler ML, McCormack DG, Parraga G: Imaging of lung function using hyperpolarized helium-3 magnetic resonance imaging: Review of current and emerging translational methods and applications. J Magn Reson Imaging 2010, 32:1398-1408.

11. Gur D, Shabason L, Borovetz HS, Herbert DL, Reece GJ, Kennedy WH, Serago C: Regional Pulmonary Ventilation Measurements by Xenon Enhanced Dynamic Computed Tomography: An Update. J Comput Assist Tomog 1981, 5:678-683.

12. Yamamoto T, Kabus S, Klinder T, Lorenz C, von Berg J, Blaffert T, Loo BW Jr, Keall PJ: Investigation of four-dimensional computed tomography-based pulmonary ventilation imaging in patients with emphysematous lung regions. Phys Med Biol 2011, 56:2279-2298.

13. Rietzel E, Pan T, Chen GTY: Four-dimensional computed tomography: Image formation and clinical protocol. Med Phys 2005, 32:874-889.

14. Ding K, Bayouth JE, Buatti JM, Christensen GE, Reinhardt JM: 4DCT-based measurement of changes in pulmonary function following a course of radiation therapy. Med Phys 2010, 37:1261-1272.

15. Yamamoto T, Kabus S, von Berg J, Lorenz C, Keall PJ: Impact of four-dimensional computed tomography pulmonary ventilation imaging-based functional avoidance for lung cancer radiotherapy. Int J Radiat Oncol Biol Phys 2011, 79:279-288.

16. Yaremko BP, Guerrero TM, Noyola-Martinez J, Guerra R, Lege DG, Nguyen $L T$, Balter PA, Cox JD, Komaki R: Reduction of normal lung irradiation in locally advanced non-small-cell lung cancer patients, using ventilation images for functional avoidance. Int J Radiat Oncol Biol Phys 2007, 68:562-571.

17. Yu H, Zhang SX, Wang RH, Zhang GQ, Tan JM: The feasibility of mapping dose distribution of 4DCT images with deformable image registration in lung. Biomed Mater Eng 2014, 24:145-153.

18. Sorzano CO, Thévenaz $P$, Unser M: Elastic registration of biological images using vector-spline regularization. IEEE Trans Biomed Eng 2005, 52:652-663.

19. Reinhardt JM, Ding K, Cao K, Christensen GE, Hoffman EA, Bodas SV: Registration-based estimates of local lung tissue expansion compared to xenon CT measures of specific ventilation. Med Image Anal 2008, 12:752-763.

20. Kim M, Lee J, Ha B, Lee R, Lee KJ, Suh HS: Factors predicting radiation pneumonitis in locally advanced non-small cell lung cancer. Radiat Oncol J 2011, 29(3):181-190.

21. Yin Y, Chen JH, Li BS, Liu TH, Lu J, Bai T, Dong XL, Yu JM: Protection of lung function by introducing single photon emission computed tomography lung perfusion image into radiotherapy plan of lung cancer. Chin med J (Engl) 2009, 122:509-513.

22. Huang TC, Hsiao CY, Chien CR, Liang JA, Shih TC, Zhang GG: IMRT treatment plans and functional planning with functional lung imaging from 4D-CT for thoracic cancer patients. Radiat Oncol 2013, 8:3.

23. Guerrero T, Sanders K, Castillo E, Zhang Y, Bidaut L, Pan T, Komaki R: Dynamic ventilation imaging from four-dimensional computed tomography. Phys Med Biol 2006, 51:777-791.

24. Guerrero T, Sanders K, Noyola-Martinez J, Castillo E, Zhang Y, Tapia R, Guerra $R$, Borghero $Y$, Komaki R: Quantification of regional ventilation from treatment planning CT. Int J Radiat Oncol Biol Phys 2005, 62:630-634.

25. Seppenwoolde Y, Muller SH, Theuws JC, Baas P, Belderbos JS, Boersma LJ, Lebesque JV: Radiation dose-effect relations and local recovery in perfusion for patients with non-small-cell lung cancer. Int J Radiat Oncol Biol Phys 2000, 47:681-690.

doi:10.1186/1748-717X-9-184

Cite this article as: Wang et al:: Optimal beam arrangement for pulmonary ventilation image-guided intensity-modulated radiotherapy for lung cancer. Radiation Oncology 2014 9:184. 